\title{
Combined introduction of anti-IL2 receptor antibodies, mycophenolic acid and tacrolimus: effect on malignancies after renal transplantation in a single-centre retrospective cohort study
}

\author{
Philippe Braconnier ${ }^{1}$, Véronique del Marmol $^{2}$, Nilufer Broeders ${ }^{1}$, Mireille Kianda ${ }^{1}$, Annick Massart ${ }^{1}$, \\ Anne Lemy ${ }^{1}$, Lidia Ghisdal ${ }^{1}$, Alain Le Moine ${ }^{1}$, Philippe Madhoun ${ }^{1}$, Judith Racapé ${ }^{1}$, Daniel Abramowicz ${ }^{1}$ \\ and Karl Martin Wissing ${ }^{1,3}$
}

\begin{abstract}
${ }^{1}$ Renal Transplantation Clinic, Department of Nephrology, ULB Hopital Erasme, Brussels, Belgium, ${ }^{2}$ Department of Dermatology, ULB Hopital Erasme, Brussels, Belgium and ${ }^{3}$ Present address: Department of Nephrology, Universitair Ziekenhuis Brussel, Brussels, Belgium

Correspondence and offprint requests to: Karl Martin Wissing; E-mail:wissing@ulb.ac.be
\end{abstract}

\begin{abstract}
Background. Several studies suggest that the introduction of tacrolimus (TRL), mycophenolic acid (MPA) and interleukin 2 receptor antibodies (IL2Ra) as single drugs more than a decade ago has not increased the risk of malignancy after renal transplantation. However, only limited data are available on their carcinogenic effects when used in combination as a potent immunosuppressive regimen.
\end{abstract}

Methods. A retrospective single-centre cohort study on 929 adult renal transplant recipients. Investigation of the effect of two consecutive immunosuppressive regimens [1993-98, $N=405$, anti-lymphocyte antibodies, cyclosporine and azathioprine (AZA); 1999-2007, $N=524$, predominantly IL2Ra, TRL and MPA] on the incidence rate of skin cancer, solid tumours and post-transplant lymphoproliferative disease (PTLD).

Results. In total, 365 malignancies developed among 113 patients. As compared to the previous cyclosporine and AZA-based immunosuppression, the introduction of the new immunosuppressive regimen did not increase the incidence rate of skin cancer [rate ratio $0.84 ; 95 \%$ confidence interval (CI) $0.48-1.46]$, solid tumours $(0.89 ; 95 \%$ CI $0.46-1.67)$ and PTLD (0.82; 95\% CI 0.28-2.21). Patients treated with the more recent regimens less frequently developed multiple skin cancers and invasive squamous cell cancer. Skin cancer after transplantation was strongly associated with the development of solid tumours (odds ratio $5.2 ; \mathrm{P}<0.0001)$. The introduction of the new immunosuppressive drugs reduced the incidence of first year acute rejection from 34.8 to $13.2 \%(\mathrm{P}<0.0001)$.

Conclusion. Although significantly more efficient in the prevention of acute rejection, the introduction of TRL, MPA and IL2Ra-based immunosuppression after kidney transplantation was not associated with an increased incidence of skin cancer, solid tumours or PTLD.
Keywords: immunosuppression; kidney transplantation; malignancy; mycophenolic acid; tacrolimus

\section{Introduction}

Immunosuppression after kidney transplantation is associated with a major increase in the risk of malignancy $[1,2]$. Skin cancer is the most commonly encountered malignancy in renal transplant recipients with up to 100 -fold increase in risk as compared to an age-adjusted general population [1]. The risk increase is constant over time and long-term cohort studies have documented a $75 \%$ incidence of skin cancer and a 33\% incidence of non-skin cancer after 30 years of transplantation [3]. Non-Hodgkin lymphoma is the second most common malignancy in kidney transplant recipients with a relative risk of up to $40 \%$ as compared to the general population [1]. Post-transplant malignancy is a cause of significant morbidity, can lead to graft loss when immunosuppression is tapered and results in cancer-related death in up to $40-50 \%$ of patients with solid tumours or lymphoma [4-6].

During the second half of the 1990s, a new standard regimen, combining induction therapy with interleukin 2 receptor antibodies (IL2Ra) (basiliximab or daclizumab), the calcineurin inhibitor tacrolimus (TRL) and the anti-metabolite agent mycophenolic acid (MPA), progressively replaced the previous combination of induction therapy with antilymphocyte antibodies such as thymoglobulin or the anti$\mathrm{CD} 3 \epsilon$ monoclonal antibody (muromonab-CD3, OKT3 ${ }^{\circledR}$ ), the calcineurin inhibitor cyclosporine and the anti-metabolite azathioprine (AZA). The introduction of this new regimen was associated with a marked improvement in the immunosuppressive efficacy and reduced the incidence of acute rejection episodes after renal transplantation to $<15 \%$ in the majority of centres $[7,8]$. The increased immunosuppressive 
effect of the new regimen has also been shown by the appearance of BK virus nephropathy, a new and previously unreported infectious complication in recipients or organ transplants [9].

The relation between immunosuppression and tumourigenesis is incompletely understood but increasing intensity of immunosuppression has been previously shown to increase the incidence of post-transplant malignancy in a prospective and controlled trial [10]. Several large registry analyses have investigated the effect of the new classes of immunosuppressants on post-transplant malignancy but did not observe an increase in non-Hodgkin lymphoma or solid tumours in transplant recipients treated with IL2Ra or MPA [1, 11-13]. However, these studies had some weaknesses: (i) most had a rather short follow-up time; (ii) outcome measures in large databases were not validated for accuracy; (iii) immunosuppressive drugs were considered individually and not as part of immunosuppressive regimens; (iv) studies did not document whether patients treated with the more recent drugs indeed received a higher immunosuppressive load as indicated by lower rates of acute rejection episodes and graft loss from rejection and (v) only very limited data are available on the impact of the new immunosuppressive drugs on skin cancer.

The objective of our study was to address these issues in a retrospective cohort study investigating the effect of the current immunosuppressive regimens on the incidence rate of post-transplant malignancies.

\section{Materials and methods}

Pre- and post-transplant data from all renal transplantations performed in our centre between January 1993 and December 2007 were extracted from a computer database, which contains routinely collected data from all patients who received a renal transplant at the University of Brussels since 1965. We excluded paediatric transplantations (recipients of $<18$ years) and combined transplantations from the current analysis. The remaining 929 adult renal transplantations constitute the study population.

\section{Post-transplant tumours}

The occurrence of tumours after transplantation was the outcome of interest. The database records the medical history of tumours before transplantation and has a yearly data entry on tumours that develop after transplantation. Data on skin tumours in the database were also crosschecked for completeness with the patient files of the dermatologist who systematically follows all transplant recipients in our centre (V.D.M.). For all tumours identified in the database, one of the investigators (P.B.) conducted a detailed review of the patient's medical records to ascertain the type of malignancy [skin cancer, solid tumour or post-transplant lymphoproliferative disease (PTLD)]; the clinical and pathological tumour classification at diagnosis according to the American Joint Committee on cancer (AJCC) TNM staging system [14]; the type of treatment (surgery, radiotherapy, chemotherapy or other) and the clinical evolution (death from the disease, death from other cause, tumour remission, evolving tumour) in the patient's medical records. Only histologically diagnosed tumours were taken into account. Skin cancers were classified as in situ squamous cell carcinomas (SCCs), invasive SCCs, basal cell carcinomas (BCCs), melanomas, Kaposi's sarcomas (skin confinement or visceral involvement) or other. According to the AJCC histopathological grading system, SCCs and BCCs were graded in well differentiated (G1), moderately differentiated (G2) or undifferentiated (G4). PTLD's were classified according to the World Health Organisation Classification (early lesions, polymorphic, monomorphic, other) [15]; localization (gastro-intestinal, pulmonary, central nervous system, kidney graft, liver or other); B- or T-cell neoplasms and Epstein-Barr virus (EBV) status. Finally, solid tumours were classified as kidney carcinoma, upper tract urothelial carcinoma, bladder carcinoma, colo-rectal carcinoma, breast carcinoma, lung carcinoma, prostate carcinoma or other. Information on post-transplant malignancies was collected until either death with a functioning graft, returning to dialysis for $>1$ month or loss to follow-up occurred.

\section{Immunosuppressive therapy}

The type of immunosuppression regimen was the exposure of interest in this cohort study. Patients received different types of immunosuppression during the 15-year follow-up. During the first period from 1993 to 1998 , the majority of patients received an immunosuppressive regimen based on induction with anti-CD3 monoclonal antibodies (muromonab-CD3, OKT3 ${ }^{\circledR}$ ), cyclosporine and AZA. During the year 1999, the three components of this regimen were progressively replaced. IL2Ra became the standard induction therapy, whereas thymoglobulin (ATG) use was restricted to patients at high immunological risk. AZA was replaced by MPA and TRL became the standard calcineurin inhibitor $(N=407$ versus 127 patients treated with the microemulsion formulation of cyclosporine). Transplantations were therefore subdivided into two successive periods of immunosuppression from 1993 to 1998 ('<1999'; $N=405$ ) and from 1999 to 2007 (' $\geq 1999$ '; $N=524$ ). All patients received methylprednisolone intravenously at the dose of 250 and $125 \mathrm{mg}$ on the day of transplantation and the first post-operative day, followed by tapered doses as maintenance therapy previously described [8].

\section{Data analysis}

Categorical and continuous variables with or without normal distribution were summarized as proportions, means with SD and medians with the interquartile range, respectively. Hypothesis testing of associations was done with the chi-squared test, $t$-test and Wilcoxon rank-sum test.

Immunological graft loss was calculated by the Kaplan-Meier method, censoring graft losses due to causes unrelated to acute or chronic rejection, such as patient death, technical failure or recurrence of primary kidney disease.

The risk of skin cancer, solid tumour and PTLD was compared by calculating the Kaplan-Meier estimates of the cumulative incidence. Due to variable follow-up times, the incidence rate of patients who develop the first tumour of interest per 1000 patient-years (py) at risk was calculated. Time at risk was defined as the delay between the date of transplantation and either time of tumour diagnosis, patient death, return to dialysis or loss to follow-up. As many patients developed multiple skin cancers, we also calculated the incidence rate of skin cancer without censoring the patient after the development of the first tumour. The effect of immunosuppression on the risk of post-transplant cancer was assessed by incidence rate ratios with $95 \%$ confidence intervals (CIs). The effect of immunosuppression on the occurrence of skin tumours after adjustment for other risk factors was assessed by multivariate negative binominal regression modelling. The effect of risk factors on the occurrence of a first tumour was calculated by using univariate and multivariate competing risk regression (CRR) modelling with the STATA stcrreg command. The proportional hazards assumption was tested using Schoenfeld residuals and by graphically displaying the plots of $\ln (-\ln (\mathrm{S}))$ against $\ln (\mathrm{t})$. Interaction between variables was tested by creating product terms that were entered into the multivariate models. The Wald test of the corresponding regression coefficient was used for hypothesis testing.

Long-term survival in patients with tumours was estimated by the Kaplan-Meier method. A bilateral P-value of $<0.05$ was used to reject the null hypothesis in all hypothesis testing. All data management and statistical analysis were realized with STATA 11.

\section{Ethical considerations}

The procedures of data collection and measures taken to maintain data confidentiality in the database of renal transplant recipients have been reviewed and approved by the ULB Hôpital Erasme Ethics committee. Patients were informed at the moment of listing for renal transplantation that their medical data will be collected in the centre and Eurotransplant databases for medical and research purposes. The protocol of the present study was reviewed and approved by the ULB Hopital Erasme ethics committee.

\section{Results}

Patients transplanted under the more recent immunosuppressive regimen were significantly older and had spent 
less time on dialysis before transplantation (Table 1). Total time at risk for the overall cohort was 5896 years with 3577 years for patients transplanted before and 2319 years for patients transplanted from 1999 onwards. About 90\% of patients received cyclosporine A (CsA) in combination with AZA in the period before 1999 and a calcineurin inhibitor in combination with MPA thereafter. Mammalian target of rapamycin (mTOR) antagonists were used in only three patients $(0.7 \%)$ before 1999 but in $11.3 \%$ of transplantations thereafter. The use of the new immunosuppressive agents was associated with a highly significant reduction in first year acute rejection episodes from 141/ $405(34.8 \%)$ to $69 / 524$ (13.2\%) [odds ratio (OR) $0.28 ; 95 \%$ CI $0.2-0.4 ; \mathrm{P}<0.0001]$. The 5-year Kaplan-Meier estimate of graft loss from rejection was reduced from 13.5 to $8 \%$ in the more recent period [hazard ratio (HR) $0.53 ; 95 \%$ CI $0.35-0.79 ; \mathrm{P}=0.002]$.

\section{Association of the new immunosuppressive regimen with the incidence of cancer after transplantation}

In the overall population, 365 malignancies developed among 113 patients (12.2\%; 95\% CI 10.1-14.3\%): 287 skin cancers $(78.6 \%), 58$ solid cancers $(15.9 \%)$ and 20 post-transplant lymphoproliferative disorders or PTLD (5.5\%). The development of at least one neoplasia after transplantation was associated with older age (48.2 \pm 11.0 versus $42.9 \pm 12.5$ years in patients without neoplasia; $\mathrm{P}<0.0001)$, a history of cancer before transplantation (7.1 versus $2.8 \% ; \mathrm{P}=0.018$ ) and Chinese herb nephropathy as primary kidney disease (11.5 versus $4.5 \%$; $\mathrm{P}=0.002)$. Treatment of acute rejection and use of mTOR antagonists were not associated with any type of cancer (data not shown).

Kaplan-Meier estimates of the cumulative incidence of skin cancer (Figure 1a), solid tumour (Figure 1b) and PTLD (Figure 1c) did not differ significantly during the two immunosuppressive periods. The Kaplan-Meier method can overestimate the cumulative incidence in the case that a large proportion of patients is censored because of competing outcomes. Univariate CRR with graft loss and patient death as competing outcomes confirmed that the hazard of malignancy was not increased in the more recent period (CRR HRs of 0.65 for skin cancer, 0.77 for solid tumour and 0.98 for PTLD, $\mathrm{P}>0.05$ for all associations). To take into account the different length of followup during the two immunosuppressive periods, rates of cancer per 1000 py at risk and the corresponding rate ratios were calculated (Table 2). Although the incidence rates of a first skin cancer, solid tumour and PTLD did not differ significantly, patients less frequently developed multiple skin cancers during the more recent period (incidence rate ratio $0.66 ; 95 \% \mathrm{CI} 0.51-0.85 ; \mathrm{P}=0.001$ ).

Multivariate analysis by negative binominal regression modelling identified age at transplantation, male gender and the immunosuppressive period as independent risk factors for post-transplant skin cancer (Table 3). Age acted as a negative confounder because adjustment for age further reduced the incidence rate ratio of skin cancer in patients receiving the new immunosuppressive regimen to $0.22(95 \%$ CI $0.09-0.54 ; \mathrm{P}=0.002)$. When the negative binominal regression model was restricted to 373 patients who received the TRL-MPA association as compared to 437 patients treated with CsA-AZA, the adjusted incidence rate ratio of skin cancer was 0.12 (95\% CI 0.04-0.4; $\mathrm{P}=0.001)$. Patient age and Chinese herb nephropathy were the only independent predictors for the development of solid tumours in the multivariate CRR model. Chinese herb nephropathy was associated with a $>4$-fold increase in the hazard of solid tumours. This was exclusively due to the known increase in urinary tract cancer [16, 17]. Twenty-one of 22 cancers that developed in the 50 patients with Chinese herb nephropathy were urinary tract cancers. The incidence of other solid tumours $(2 \%)$ was comparable to patients with other nephropathies (3\%).

\section{Association of the new immunosuppressive regimens with the stage and clinical evolution of post-transplant tumours}

Skin cancer. Most of the skin cancers were SCCs $(n=$ $178 ; 62 \%)$, either in situ $(n=130 ; 45.3 \%)$ or invasive

Table 1. Main characteristics of kidney transplant recipients during two successive immunosuppressive periods

\begin{tabular}{|c|c|c|c|}
\hline Characteristics & $<1999(N=405)$ & $\geq 1999(N=524)$ & $\mathrm{P}$ \\
\hline Male gender & $253(62.5 \%)$ & $326(62.2 \%)$ & 0.94 \\
\hline Age at transplantation (years) ${ }^{\mathrm{a}}$ & $39.4 \pm 10.9$ & $46.8 \pm 12.6$ & $<0.0001$ \\
\hline Retransplantation & $75(18.5 \%)$ & $109(20.8 \%)$ & 0.38 \\
\hline Duration of dialysis (months) ${ }^{\mathrm{b}}$ & $33.5(19.4-58.0)$ & $27.9(13.6-49.6)$ & 0.002 \\
\hline First-year acute rejection & $141(34.8 \%)$ & $69(13.2)$ & $<0.0001$ \\
\hline History of cancer & $11(2.7 \%)$ & $20(3.8 \%)$ & 0.35 \\
\hline Splenectomy & $15(3.7 \%)$ & $20(3.8 \%)$ & 0.93 \\
\hline Chinese herb nephropathy & $34(8.4 \%)$ & $16(3.1 \%)$ & $<0.0001$ \\
\hline Follow-up time (years) ${ }^{\mathrm{b}}$ & $8.8(5.0-12.5)$ & $4.4(2.3-6.5)$ & $<0.0001$ \\
\hline \multicolumn{4}{|l|}{ Immunosuppressive agents $^{\mathrm{c}}$} \\
\hline ATG-OKT3/IL2Ra/none (\%) & $89.9 / 0 / 11.1$ & $21.6 / 57.3 / 21.2$ & $<0.0001$ \\
\hline AZA/MPA (\%) & $88.4 / 10.6$ & $0.4 / 91.2$ & $<0.0001$ \\
\hline CsA/TRL (\%) & $95.1 / 4.2$ & $22.1 / 72.0$ & $<0.0001$ \\
\hline Sirolimus/everolimus (\%) & $0.7 / 0$ & $8.6 / 2.7$ & $<0.0001$ \\
\hline
\end{tabular}

${ }^{\mathrm{a}}$ Mean $\pm \mathrm{SD}$.

${ }^{\mathrm{b}}$ Median (interquartile range)

${ }^{\mathrm{c}}$ According to intention to treat. 

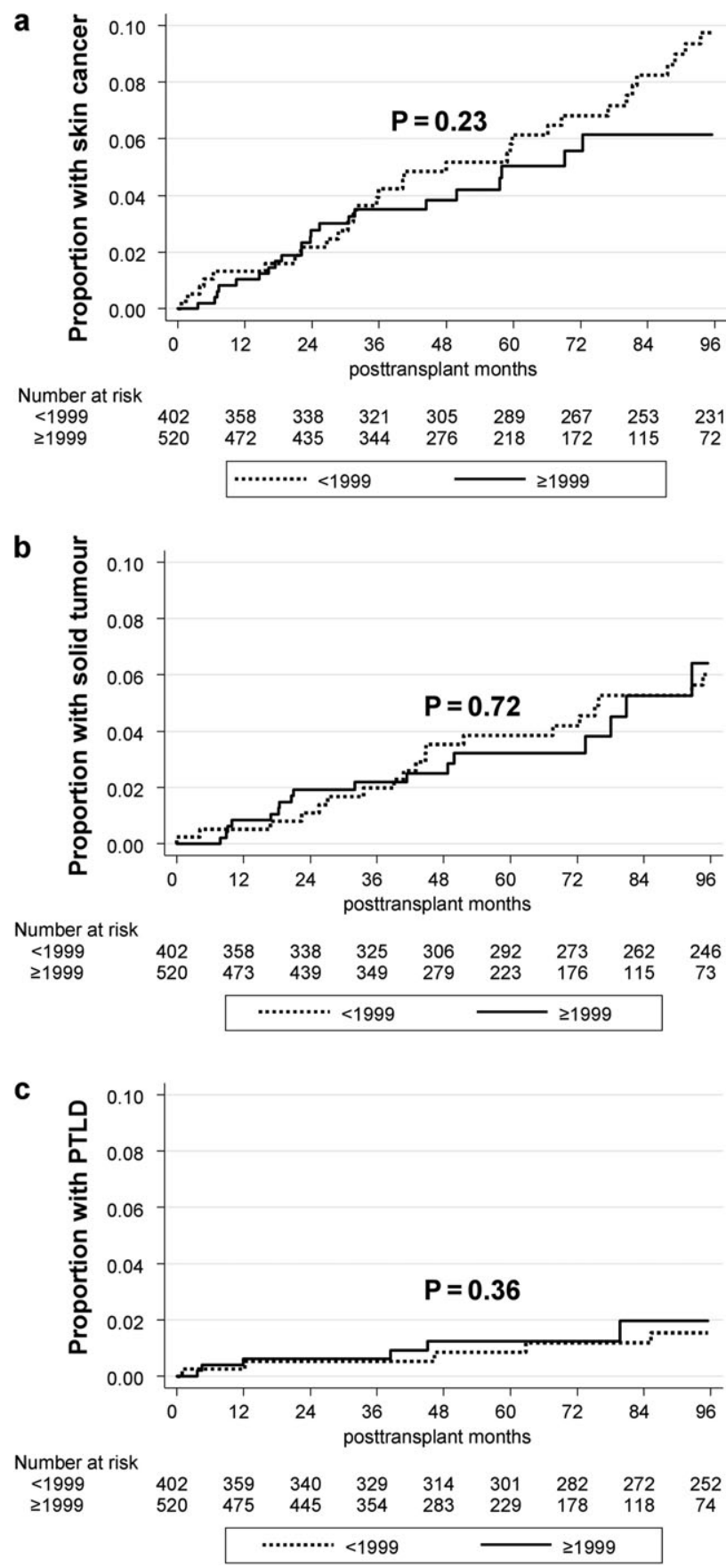

Fig. 1. Kaplan-Meier estimates of the cumulative incidence of cancer by immunosuppressive period up to 96 months after transplantation. Cumulative incidence of skin cancer (a), solid tumours (b) and post-transplant lymphoproliferative disease (c). Population at risk during the follow-up is tabulated below the graphs. Hypothesis testing by the log-rank test with $\mathrm{P}>0.05$ for all comparisons.

$(n=48 ; 16.7 \%) ; \sim 80 \%(n=144 ; 80.9 \%)$ were well differentiated on histological grading, whereas $19.1 \%(n=34)$ were moderately differentiated or undifferentiated. BCCs were less common $(n=80 ; 27.9 \%)$ with a ratio of SCCs to BCCs of 2.2:1. The median number of tumours in patients with skin cancer per patient was 2 (range 1-43). The distribution of squamous cell skin cancers according to the immunosuppressive regimen is shown in Table 4. A higher proportion of SCCs that developed in patients treated with CsA-AZA were invasive $(31.5$ versus $14.6 \%$; $\mathrm{P}=0.02)$. Among the 17 patients who developed one or several invasive SCCs, 3 died of a metastatic tumour infiltration (17.6\%). In total, $18 \%(11 / 61)$ of patients with skin cancer also developed a solid cancer compared to only $4 \%(33 / 868)$ of patients without skin cancer (OR 5.2; 95\% CI 2.5-10.9; P < 0.0001).

A total of 14 patients developed Kaposi's sarcoma and 4 patients melanoma without significant differences in stage at diagnosis and evolution between the two immunosuppressive periods.

Solid cancer. The most common solid cancers after kidney transplantation $(n=58)$ were bladder urothelial carcinoma $(n=18 ; 31 \%)$, upper-tract urothelial carcinoma $(n=8 ; 13.8 \%)$, colo-rectal cancer $(n=4 ; 6.9 \%)$, breast cancer $(n=4 ; 6.9 \%)$, kidney cancer $(n=3 ; 5.2 \%)$ and lung cancer $(n=3 ; 5.2 \%)$ (Supplentary table 1$)$. Most solid tumours were diagnosed at a Stages I-II AJCC $(n=37$; $63.8 \%)$, especially bladder urothelial carcinoma $(n=15$; $83.3 \%)$ and upper-tract urothelial carcinoma $(n=7$; $87.5 \%$ ) which might be related to systematic screening cystoscopies and bilateral ureteronephrectomy in kidney transplant recipients with Chinese herb nephropathy [16]. Around $36 \%(n=21 ; 36.2 \%)$ were detected at a late Stages III-IV, mostly colo-rectal cancer $(n=3 ; 75 \%)$, lung cancer $(n=2 ; 66.7 \%)$ and stomach cancer $(n=2 ; 100 \%)$. Overall, $24.6 \%$ of all solid tumours were diagnosed at a metastatic stage (Stage IV).

There were no significant differences between the number of solid cancer cases diagnosed at a late Stages III-IV AJCC TNM staging comparing the previous $(35.1 \%)$ and more recent immunosuppressive periods $(38.9 \%)(\mathrm{P}=0.786)$.

Among the 46 patients who developed 58 solid tumours, in total, 20 patients died of the disease (43.5\%), 19 patients were in tumour remission (41.3\%), 5 patients had an evolving tumour $(10.9 \%)$ and 2 patients died of another cause (4.3\%). Patient survival with solid cancer was $50.2 \%$ (95\% CI $33-65 \%)$ at 5 years and $40.2 \%(95 \%$ CI $22-57 \%)$ at 10 years. Survival after development of a solid tumour tended to be lower in the more recent period [5-year survival of $48.1 \%$ (17.4-73.6) versus 73.5\% (46.8-88.2); $\mathrm{P}=0.09$ ].

PTLD. The PTLD $(n=20)$ occurred in the gastro-intestinal tract in $45 \%$, the central nervous system in $20 \%$ and the renal graft in $15 \%$ of the patients. Active replication of EBV was detected by polymerase chain reaction in $69.2 \%$ of 13 tested patients. B-cell lymphomas accounted for the great majority of PTLD in the renal transplant population (93.8\%). Monomorphic (53.3\%) and polymorphic (46.7\%) PTLD were about equally frequent. Eight patients were treated successfully $(40 \%)$, eight patients died of the disease $(40 \%)$, three patients had an evolving tumour $(15 \%)$ and one patient died of another cause (5\%). Patient survival with PTLD was $41.6 \%(95 \%$ CI $14-67 \%)$ at 5 years. There were no significant differences in the incidence rate of monomorphic or polymorphic PTLD, detection of EBV and clinical outcomes between the two periods of immunosuppression. 
Table 2. Incidence rates of malignancies after kidney transplantation during two successive immunosuppressive periods

\begin{tabular}{lcccc}
\hline Incidence rate $^{\mathrm{a}}$ & $<1999$ & $\geq 1999$ & IRR $^{\mathrm{b}}(95 \% \mathrm{CI})$ & $\mathrm{P}$ \\
\hline Skin cancer $^{\mathrm{c}}$ & 11.5 & 9.7 & $0.84(0.48-1.46)$ & 0.53 \\
Total number of skin cancers $^{\mathrm{d}}$ & 56.2 & 37.1 & $0.66(0.51-0.85)$ & $0.89(0.46-1.67)$ \\
Solid tumour $^{\mathrm{c}}$ & 8.4 & 7.4 & $0.82(0.28-2.21)$ & 0.71 \\
PTLD $^{\mathrm{c}}$ & 3.7 & 3.0 & 0.94 \\
\hline
\end{tabular}

${ }^{\mathrm{a}} \mathrm{n} / 1000$ patient-years at risk.

${ }^{\mathrm{b}}$ Incidence rate ratio.

${ }^{\mathrm{c}}$ Rate of patients developing first tumour. Time at risk censored after occurrence of first tumour of interest.

${ }^{\mathrm{d}}$ Taking into account multiple tumours developing in the same patient.

Table 3. Multivariate analysis of risk factors for skin cancer and solid cancers

\begin{tabular}{|c|c|c|c|c|}
\hline \multirow[b]{2}{*}{ Risk factors } & \multicolumn{2}{|l|}{ Skin cancers ${ }^{\mathrm{a}}$} & \multicolumn{2}{|l|}{ Solid tumour ${ }^{\mathrm{b}}$} \\
\hline & IRR $(95 \%$ CI) & $\mathrm{P}$ & HR $(95 \%$ CI) & $\mathrm{P}$ \\
\hline Age at transplant (per year) & $1.11(1.06-1.16)$ & $<0.0001$ & $1.03(1.00-1.06)$ & 0.016 \\
\hline Male gender & $2.81(1.09-7.24)$ & 0.001 & $1.08(0.54-2.20)$ & 0.83 \\
\hline History of cancer & $0.53(0.04-7.35)$ & 0.64 & $2.06(0.76-5.58)$ & 0.154 \\
\hline Chinese herb nephropathy & $1.11(0.20-6.10)$ & 0.424 & $4.8(2.14-10.75)$ & $<0.0001$ \\
\hline Immunosuppressive period $\geq 1999$ & $0.22(0.09-0.54)$ & 0.002 & $0.71(0.38-1.37)$ & 0.29 \\
\hline
\end{tabular}

${ }^{a}$ Effect of risk factors on incidence rate of skin cancers modelled by negative binominal regression; IRR, incidence rate ratio.

${ }^{b}$ Effect of risk factors on the time to occurrence of first tumour modelled by competing risk regression.

Table 4. SCC type and grade of differentiation

\begin{tabular}{lrrr}
\hline SCC type & $<1999$ & $\geq 1999$ & $\mathrm{P}$ \\
\hline SCC in situ & $89(68.5 \%)$ & $41(85.4 \%)$ & \\
SCC invasive & $41(31.5 \%)$ & $7(14.6 \%)$ & 0.024 \\
$\begin{array}{l}\text { Grade of differentiation } \\
\quad \text { Well differentiated (G1) }\end{array}$ & $110(84.6 \%)$ & $34(70.8 \%)$ & \\
$\quad \begin{array}{l}\text { Moderately differentiated or } \\
\text { undifferentiated (G2 or G4) }\end{array}$ & $20(15.4 \%)$ & $14(29.2 \%) 0$ & 0.052 \\
Total & 130 & 48 & \\
\hline
\end{tabular}

\section{Discussion}

The present study shows that introduction of the current immunosuppressive regimens based on calcineurin inhibitors, MPA and induction therapy with IL2Ra antibodies did not increase the incidence rate of skin cancer, solid tumours or PTLD. Our observations are in accordance with several previous reports which documented no increase in the risk of PTLD and solid tumours by IL2Ra or MPA in recipients of solid organ transplants $[1,12,13]$. The present report complements these papers by focussing not on individual immunosuppressive agents but the effect of their combined use as what has become the current gold standard of immunosuppression [7]. By confirming in the present series that the newer drugs effectively provide more efficient immunosuppression in terms of acute rejection episodes and graft loss from rejection, our data challenge the commonly held belief that the risk of cancer is directly related to the overall immunosuppressive load.

In agreement with previous reports, the present study confirms that renal transplant recipients frequently develop multiple and invasive non-melanoma skin cancers [18-21] and that invasive SCC is complicated by tumour general- ization and death in a significant proportion of patients $[21,22]$. Furthermore, the development of skin cancer could be a marker for an increased risk to develop a solid tumour, as patients with skin cancers had a five times increased risk of developing a solid tumour. Interestingly, the incidence rate of multiple and invasive non-melanoma skin cancers was significantly lower during the more recent immunosuppressive period. The use of mTOR antagonists in a small proportion of patients during the more recent period is unlikely to explain the effect as it was also observed when the analysis was restricted to patients treated with TRL and MPA. Withdrawal of AZA from the current regimen could be instrumental because the drug generates mutagenic oxidative DNA lesions by enhancing the effect of ultraviolet A light [23]. A protective effect of MPA has also been suggested by a lower incidence of skin cancer in patients taking $3 \mathrm{~g}$ instead of $2 \mathrm{~g}$ of MPA per day [24] and by an inhibitory effect of MPA on tumour cell growth and angiogenesis in vitro [25].

mTOR antagonists inhibit tumour cell proliferation and angiogenesis and are associated with a lower incidence of cancer after organ transplantation [26, 27]. Conversion to mTOR antagonists has reduced the incidence of nonmelanoma skin cancer in high-risk patients [28] but this approach might be unsafe in patients with suboptimal graft function or proteinuria [29]. Our data suggest that conversion from AZA to MPA might be an alternative option in this category of patients.

Our cohort has a high incidence of urinary tract cancer due to the consumption of Chinese herb preparations containing Aristolochia species that dramatically increase the risk of urothelial carcinoma $[16,17]$. The present cohort with a longterm follow-up confirmed that the carcinogenic effect of aristolochic acid is limited to urinary tract tumours and does not affect the incidence of other solid tumours or skin cancer. 
Twenty of the 929 patients in our cohort developed PTLD after transplantation. Previous large registry studies had reported a reduced incidence of PTLD with the use of IL2Ra instead of ATG [12, 30], MPA [12] and cyclosporine as compared to TRL [11]. We were unable to detect an effect of immunosuppression on the incidence of PTLD. This could reflect a lack of association as previously reported [13, 31] or be related to the relatively low incidence rate and insufficient statistical power to detect an association in our cohort.

Our study has several limitations. Firstly, potential risk factors for cancer, such as smoking, sun exposure, and a positive family history could not be assessed in the present retrospective dataset. It is, however, unlikely that these risk factors have changed substantially during the two consecutive periods. Secondly, data were analysed on an intention-to-treat basis that does not take into account subsequent changes in immunosuppressive agents. Differences in follow-up between patients treated with successive immunosuppressive regimens might interfere with the estimation of rates of cancer. We addressed this issue by calculating incidence rates taking into account time at risk and by using competing risk analysis because of censoring for competing outcomes. In spite of these precautions, we cannot completely rule out that the higher incidence of multiple and recurring skin cancers under the previous regimen is caused by more prolonged exposure to immunosuppression. These observations therefore have to be confirmed after a longer follow-up of patients treated with the more recent regimen. Finally, we identified age as a positive confounder during the more recent period but cannot rule out that measures of effect remain confounded by unidentified risk factors.

In conclusion, this study shows that the current immunosuppressive regimen based on TRL, MPA and IL2Ra induction therapy is not associated with an increased incidence rate of malignancy as compared to the previous regimen in spite of improved prevention of graft rejection. Furthermore, the current regimen is associated with a marked reduction in the incidence rate of skin cancer. This suggests that immunosuppressive regimens can differentially affect immunosuppressive mechanisms involved in graft rejection and tumour surveillance.

\section{Supplementary data}

Supplementary data are available online at http:// ndt.oxfordjournals.org.

Acknowledgements. The excellent work of our research nurses Mrs Brigitte Borré, Françoise Bernard, Sylvie Arias, Nicole Liétaer and Anne Killens is gratefully acknowledged. P.B. has participated in setting up the study protocol, data collection and interpretation of the results and in the writing of the paper. J.R. has participated in data collection, data analysis and writing of the paper. V.D. has participated in data collection, interpretation of the results and writing of the paper. N.B., M.K., A.M., A.L., L.G., A.L.M. and P.M. have participated in the follow-up of the patients, data collection and the writing of the paper. D.A. has participated in the development of the research question and the study protocol, interpretation of the results and the writing of the paper. K.M.W. has participated in the development of the research question, setting up the study protocol, data collection, data analysis and writing of the paper.
Transparency declarations. None declared.

Conflict of interest statement. None declared.

\section{References}

1. Kasiske BL, Snyder JJ, Gilbertson DT et al. Cancer after kidney transplantation in the United States. Am J Transplant 2004; 4: 905-913

2. Vajdic CM, McDonald SP, McCredie MR et al. Cancer incidence before and after kidney transplantation. JAMA 2006; 296: 2823-2831

3. Sheil AG. Patterns of malignancies following renal transplantation. Transplant Proc 1999; 31: 1263-1265

4. Kauffman HM, Cherikh WS, McBride MA et al. Post-transplant de novo malignancies in renal transplant recipients: the past and present. Transpl Int 2006; 19: 607-620

5. Pedotti P, Cardillo M, Rossini G et al. Incidence of cancer after kidney transplant: results from the North Italy transplant program. Transplantation 2003; 76: 1448-1451

6. Tremblay F, Fernandes M, Habbab F et al. Malignancy after renal transplantation: incidence and role of type of immunosuppression. Ann Surg Oncol 2002; 9: 785-788

7. Ekberg H, Tedesco-Silva H, Demirbas A et al. Reduced exposure to calcineurin inhibitors in renal transplantation. N Engl J Med 2007; 357: $2562-2575$

8. Wissing KM, Fomegne G, Broeders $\mathrm{N}$ et al. HLA mismatches remain risk factors for acute kidney allograft rejection in patients receiving quadruple immunosuppression with anti-interleukin-2 receptor antibodies. Transplantation 2008; 85: 411-416

9. Dall A, Hariharan S. BK virus nephritis after renal transplantation. Clin J Am Soc Nephrol 2008; 3 (Suppl 2): S68-S75

10. Dantal J, Hourmant M, Cantarovich D et al. Effect of long-term immunosuppression in kidney-graft recipients on cancer incidence: randomised comparison of two cyclosporin regimens. Lancet 1998; 351: 623-628

11. Opelz G, Dohler B. Lymphomas after solid organ transplantation: a collaborative transplant study report. Am J Transplant 2004; 4: 222-230

12. Cherikh WS, Kauffman HM, McBride MA et al. Association of the type of induction immunosuppression with posttransplant lymphoproliferative disorder, graft survival, and patient survival after primary kidney transplantation. Transplantation 2003; 76: 1289-1293

13. Robson R, Cecka JM, Opelz G et al. Prospective registry-based observational cohort study of the long-term risk of malignancies in renal transplant patients treated with mycophenolate mofetil. Am J Transplant 2005; 5: 2954-2960

14. Greene F, Page D, Fleming I et al. AJCC Cancer Staging Manual. 6th edn. New York, NY: Springer, 2002

15. Harris NL, Jaffe ES, Diebold J et al. The World Health Organization classification of neoplasms of the hematopoietic and lymphoid tissues: report of the clinical Advisory committee meeting-Airlie House, Virginia, November, 1997. Hematol J 2000; 1: 53-66

16. Nortier JL, Martinez MC, Schmeiser HH et al. Urothelial carcinoma associated with the use of a Chinese herb (Aristolochia fangchi). N Engl J Med 2000; 342: 1686-1692

17. Lemy A, Wissing KM, Rorive S et al. Late onset of bladder urothelial carcinoma after kidney transplantation for end-stage aristolochic acid nephropathy: a case series with 15 -year follow-up. Am J Kidney Dis 2008; 51: 471-477

18. Euvrard S, Kanitakis J, Decullier E et al. Subsequent skin cancers in kidney and heart transplant recipients after the first squamous cell carcinoma. Transplantation 2006; 81: 1093-1100

19. Tessari G, Naldi L, Boschiero L et al. Incidence and clinical predictors of a subsequent nonmelanoma skin cancer in solid organ transplant recipients with a first nonmelanoma skin cancer: a multicenter cohort study. Arch Dermatol 2010; 146: 294-299

20. Lott DG, Manz R, Koch C et al. Aggressive behavior of nonmelanotic skin cancers in solid organ transplant recipients. Transplantation 2010; 90: 683-687 
21. Mackenzie KA, Wells JE, Lynn KL et al. First and subsequent nonmelanoma skin cancers: incidence and predictors in a population of New Zealand renal transplant recipients. Nephrol Dial Transplant 2010; 25: 300-306

22. Joseph MG, Zulueta WP, Kennedy PJ. Squamous cell carcinoma of the skin of the trunk and limbs: the incidence of metastases and their outcome. Aust N Z J Surg 1992; 62: 697-701

23. O'Donovan P, Perrett CM, Zhang X et al. Azathioprine and UVA light generate mutagenic oxidative DNA damage. Science 2005; 309: 1871-1874

24. Mathew TH. A blinded, long-term, randomized multicenter study of mycophenolate mofetil in cadaveric renal transplantation: results at three years. Tricontinental Mycophenolate Mofetil Renal Transplantation Study Group. Transplantation 1998; 65: 1450-1454

25. Koehl GE, Wagner F, Stoeltzing O et al. Mycophenolate mofetil inhibits tumor growth and angiogenesis in vitro but has variable antitumor effects in vivo, possibly related to bioavailability. Transplantation 2007; 83: 607-614

26. Guba M, Graeb C, Jauch KW et al. Pro- and anti-cancer effects of immunosuppressive agents used in organ transplantation. Transplantation 2004; 77: 1777-1782
27. Campistol JM. Minimizing the risk of posttransplant malignancy. Transplantation 2009; 87: S19-S22

28. Salgo R, Gossmann J, Schofer H et al. Switch to a sirolimus-based immunosuppression in long-term renal transplant recipients: reduced rate of (pre-)malignancies and nonmelanoma skin cancer in a prospective, randomized, assessor-blinded, controlled clinical trial. Am $J$ Transplant 2010; 10: 1385-1393

29. Schena FP, Pascoe MD, Alberu J et al. Conversion from calcineurin inhibitors to sirolimus maintenance therapy in renal allograft recipients: 24-month efficacy and safety results from the CONVERT trial. Transplantation 2009; 87: 233-242

30. Opelz G, Naujokat C, Daniel V et al. Disassociation between risk of graft loss and risk of non-Hodgkin lymphoma with induction agents in renal transplant recipients. Transplantation 2006; 81: 1227-1233

31. Webster AC, Woodroffe RC, Taylor RS et al. Tacrolimus versus ciclosporin as primary immunosuppression for kidney transplant recipients: meta-analysis and meta-regression of randomised trial data. BMJ 2005; 331: 810

Received for publication: 9.2.11; Accepted in revised form: 26.9.11 